\title{
Alteration of tumor associated neutrophils by PIK3CA expression in endometrial carcinoma from TCGA data
}

\author{
Yinglian Pan', Li Ping Jia², Yuzhu Liu², Yixu Han² and Qingchun Deng ${ }^{2 *}$
}

\begin{abstract}
Uterine corpus endometrial carcinoma (UCEC) is one of the most common cancer in female worldwide. PIK3CA has been proven to be a strong prognostic biomarker in UCEC. Nevertheless, current studies have not investigated what effects PIK3CA had on tumor associated neutrophils (TANss). Kaplan-Meier methods were used to compute the survival time of TCGA UCEC patients. GO and KEGG enrichment analysis unveiled relevant pathways PIK3CA affected using DEGs between PIK3CA high expression group and PIK3CA low expression group in TCGA UCEC, as well as GSEA. immune infiltration status was calculated using TIMER. We found that PIK3CA influenced a number of pathways including immune related pathways. The fraction of TANs was certainly altered by PIK3CA expression in UCEC. Our findings suggest that PIK3CA expression may play an important role in tumor immune microenvironment and could alter fraction of TANs in UCEC.
\end{abstract}

Keywords: Uterine corpus endometrial carcinoma, PIK3CA, Tumor associated neutrophils

\section{Introduction}

Uterine corpus endometrial carcinoma (UCEC) is the most common gynecologic malignancy in plenty of countries, 9 out of 10 women with early-stage disease present with the symptom of postmenopausal bleeding [1]. Over 50,000 women die from UCEC every year in the world [2]. UCSC disrupts a lot of signal pathways including PI3K/ PTEN/AKT/mTOR pathway [3]. PI3K pathway serves a pivotal function that may have potential for defining targeted therapy for the treatment of grade 3 UCEC [4]. The main component of PI3K pathway, PIK3CA, is a strong prognostic biomarker in UCEC and associates with disease-specific mortality [5]. PIK3CA missense mutation is associated with unfavorable outcome in endometrioid carcinoma [6, 7]. PIK3CA and PIK3R1 mutations were frequent and showed a strong tendency for mutual exclusivity in UCSC [8]. The previous work on molecular mechanism of UCEC has indicated that PIK3CA played a crucial role in development of tumor.

\footnotetext{
* Correspondence: qingchun0503@163.com

${ }^{2}$ Department of Gynecology, The Second Affiliated Hospital of Hainan Medical University, Haikou, Hainan 570102, People's Republic of China Full list of author information is available at the end of the article
}

Tumor-associated neutrophils (TANs) are phenotypically distinct from circulating neutrophils in terms of their surface protein composition and cytokines/chemokine activity, modulate the tumor immune microenvironment [9]. TANs can perform pro-tumoral functions, strengthening tumor cell invasion and metastasis, angiogenesis, and extracellular matrix remodeling, while inhibiting the antitumoral immune surveillance [10]. TANs recruit macrophages and Treg cells to hepatocellular carcinoma to promote their growth, progression, and resistance to sorafenib [11], which implied the regulated role of TANs in tumor immune microenvironment. High levels of TANs have been associated with a poor prognosis in different malignances [12]. TANs are an important component of the immune cell infiltrate in colorectal cancer and assessment of TAN infiltration may help identify patients likely to benefit from 5-FUbased chemotherapy [13]. Furthermore, TANs can also inhibit metastatic seeding in the lung cancer through hydrogen peroxide generation [14]. In general, the significant function of TANs has been verified in various researches.

However, until now no field research on what effects and internal mechanism PIK3CA has on TANs in UCEC 
have been reported. On the basis of existing literature data, we carried out studies in an effort to clarify the crucial role of PIK3CA in UCEC immune microenvironment.

\section{Results}

\section{Expression level of PIK3CA was correlated with survival} time of UCEC patients

The Human Protein Atlas showed the expression of PIK3CA in UCEC varied from medium to high and mainly in cytoplasm (Fig. 1a). Kaplan-Meier Survival Analysis [15] of 542 The Cancer Genome Atlas (TCGA) UCEC patients grouped by the expression of PIK3CA revealed a poorer clinical outcome in patients with high PIK3CA expression compared to those with low PIK3CA expression $(P=0.0018)$ (Fig. 1b). There were 170 mutation sites in TCGA UCEC patients PIK3CA gene analyzed by cBioPortal [16, 17], including 161 missense sites, 8 inframe sites, and 1 truncating sites (Fig. 1c). Patients without alteration in PIK3CA had poorer clinical outcome than those with alteration $(P=0.0115)$ (Fig. 1d). PIK3CA expression could influence survival time of UCEC patients, which suggested its prognosis.

\section{A lot of molecules were related to PIK3CA expression in UCEC}

We divided TCGA UCEC patients into two subgroups by PIK3CA relative expression, PIK3CA low group $(n=$ $255)$ and PIK3CA high group $(n=256)$. By setting $\log _{2}(-$ Fold change) as \pm 1 and FDR as 0.01 , we identified 4315 Different Expressing Genes (DEGs) between PIK3CA low group and PIK3CA high group (Fig. 2a). There were 3623 upregulated DEGs and 692 downregulated DEGs.

There were a variety of genes that expressed dissimilarly between PIK3CA low group and PIK3CA high group, which implied these genes played significant roles in the development of UCEC through PIK3CA (Fig. 2c, d). In addition, a number of genes got clustered and collectively expressed higher in the majority of PIK3CA high group patients (Fig. 2c). This phenomenon showed that a set of genes may get together to influence PIK3CA expression.

\section{GO and KEGG enrichment analysis unveiled pathways PIK3CA affected in UCEC}

In order to find pathways PIK3CA affects, we applied GO and KEGG enrichment analysis using top 500 DEGs (Fig. 2b).

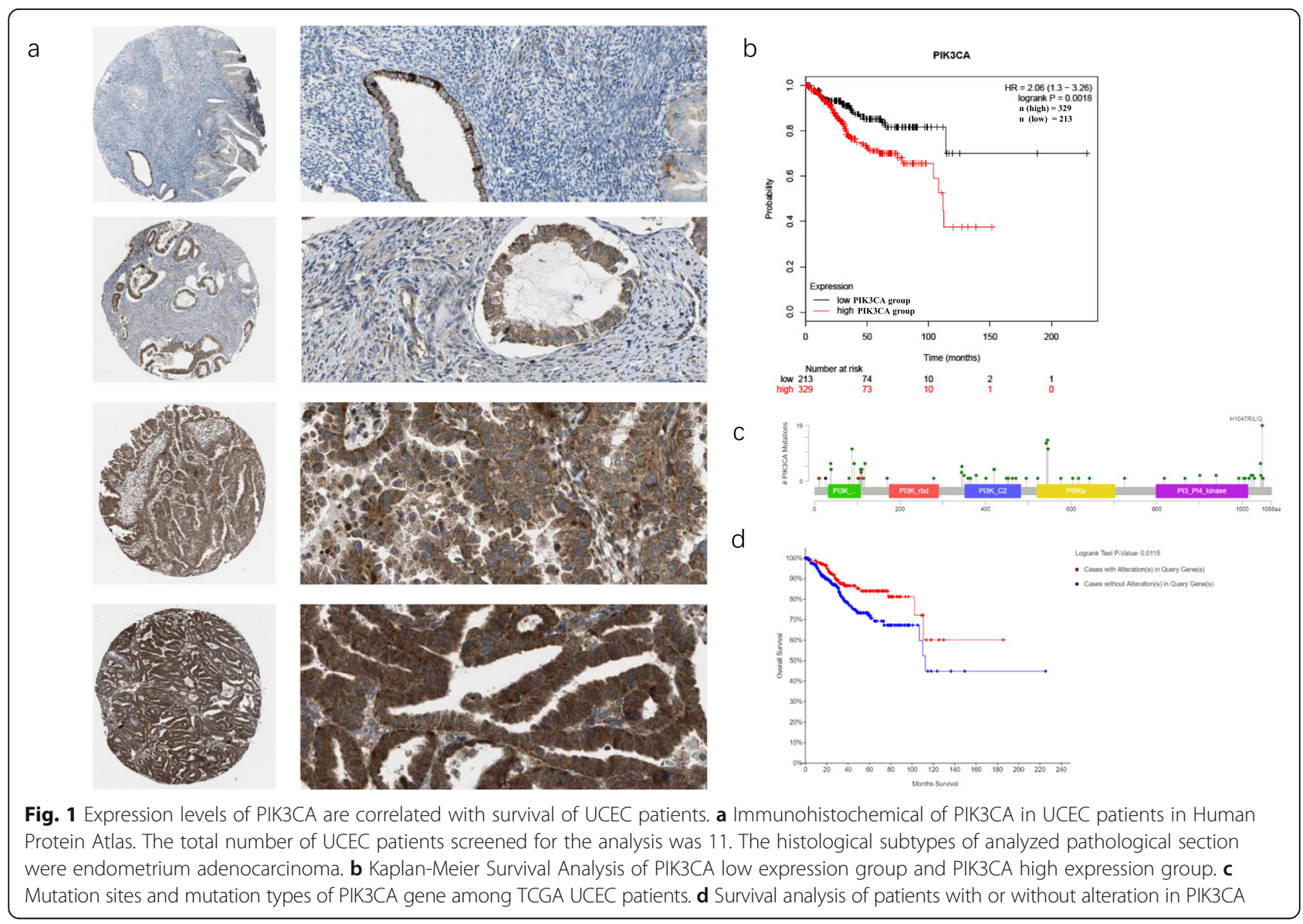




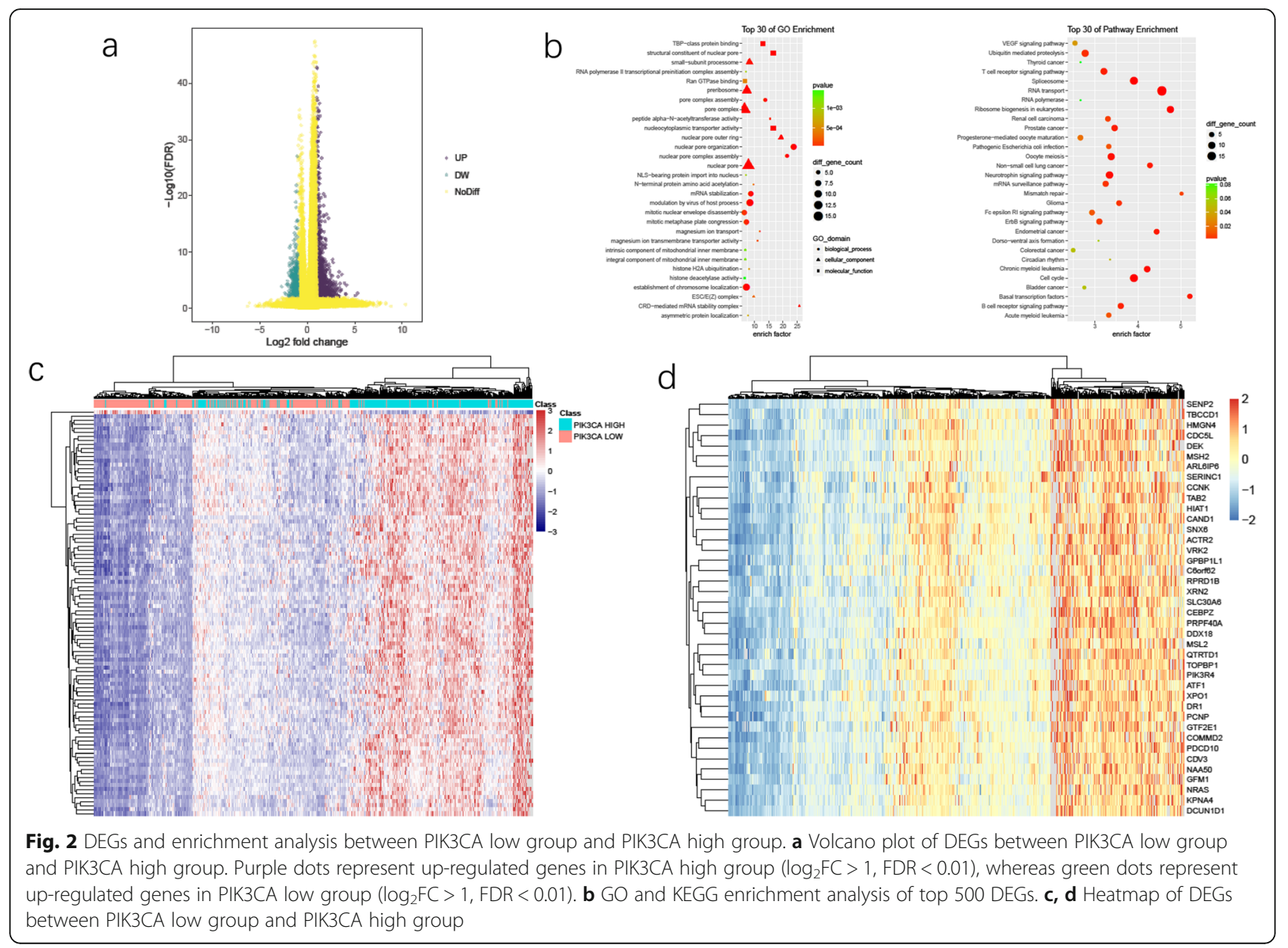

GO enrichment analysis showed plenty of pathways were well enriched, including CRD-mediated mRNA stability complex, nuclear pore organization, nuclear pore complex assembly, nuclear pore outer ring, structural constituent of nuclear pore, nucleocytoplasmic transporter activity, peptide alpha- $\mathrm{N}$-acetyltransferase activity, pore complex assembly, TBP-class protein binding, magnesium ion transport (Fig. 2b, left). Most of these pathways belonged to biological process and cellular component related pathways.

KEGG enrichment analysis showed some pathways were enriched significantly, including Basal transcription factors, Mismatch repair, Ribosome biogenesis in eukaryotes, RNA transport, Endometrial cancer, Non-small cell lung cancer, Chronic myeloid leukemia, Cell cycle, Spliceosome (Fig. 2b, right). PIK3CA expression could affect multiple tumor progression as reported [18-20]. Besides, B cell receptor signaling pathway $(P=0.002$, enrich factor $=3.6)$, $\mathrm{T}$ cell receptor signaling pathway $(P=0.001$, enrich factor $=3.21)$ and Natural killer cell mediated cytotoxicity $(P=0.06$, enrich factor $=1.98)$ were well enriched, which suggested PIK3CA expression might have an effect on immune system.
In addition, top 800 DEGs KEGG enrichment analysis showed another immune related pathway, mTOR signaling pathway, was significant enriched $(P=0.013$, enrich factor $=4.2)$ (Additional file 1: Figure S1).

\section{PIK3CA expression significantly influenced neutrophil related pathway}

Next, we utilize Gene Set Enrichment Analysis (GSEA) to enrich in immune related pathways using PIK3CA low group and PIK3CA high group expression data. The $\mathrm{T}$ Cell Receptor plays a key role in the immune system. As can be seen from Fig. 3a, PIK3CA high group was enriched in T Cell Receptor Signaling Pathway (NES = $1.77, \mathrm{FDR}=0.0034$ ), which is consistent with the observation from KEGG enrichment analysis (Fig. 2b). It must also be mentioned that PIK3CA high group enriched significantly in two neutrophil related pathways $(\mathrm{NES}=$ $1.39 \mathrm{FDR}=0.0422, \mathrm{NES}=1.97 \mathrm{FDR}=0$, respectively) (Fig. 3b, c). Identical result were obtained in TGF $\beta$ pathway $(\mathrm{NES}=2.04, \mathrm{FDR}=0$ ) (Fig. $3 \mathrm{~d}$ ), which plays an important role in tumor initiation and progression and increases neutrophil-attracting chemokines resulting in recruitment and activation of neutrophils with an antitumor 
a

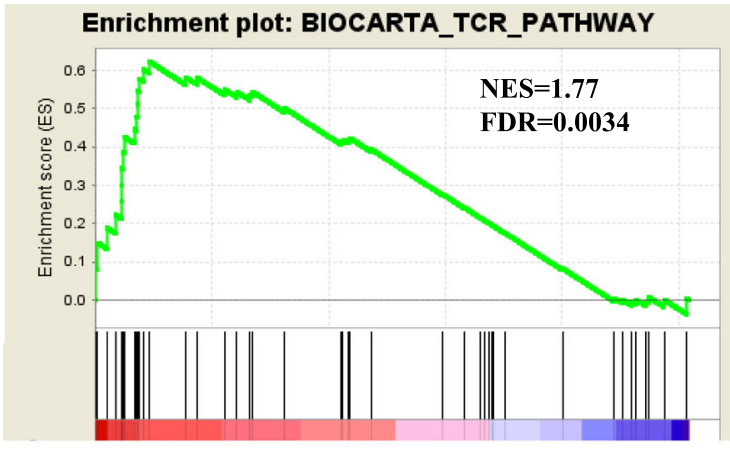

C

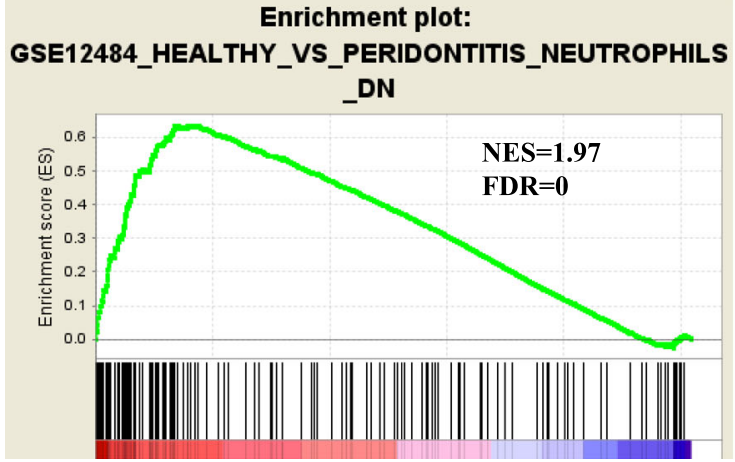

b

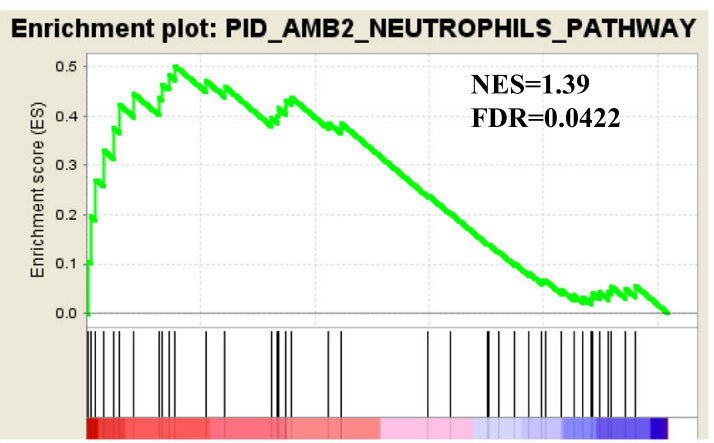

d

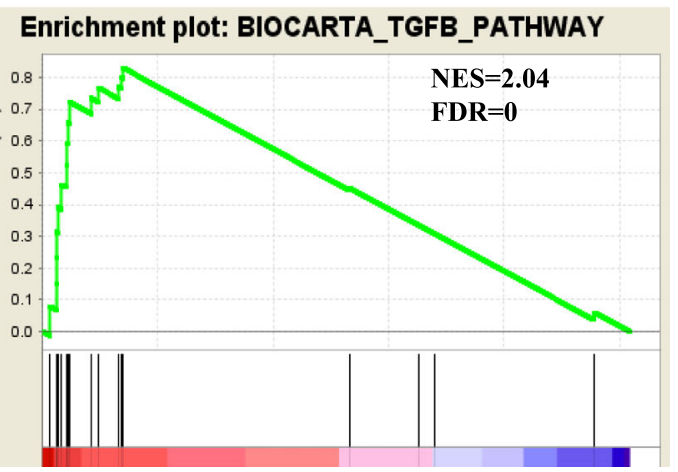

Fig. 3 GSEA analyzing PIK3CA low group and PIK3CA high group expression data in four pathways including (a) BIOCARTA TCR PATHWAY, (b) PID AMB2 NEUTROPHILS PATHWAY, (c) GSE12484 HEALTHY VS PERIDONTITIS NEUTROPHILS DN and (d) BIOCARTA TGFB PATHWAY

phenotype [21]. These results provide substantial evidence for the above assumptions that PIK3CA expression might have an effect on immune system, more probably, on TANs.

By the way, two famous tumor related pathways were well enriched, Wnt Pathway $(\mathrm{NES}=1.89, \mathrm{FDR}=0.0001)$ and JNK MAPK Pathway $(\mathrm{NES}=2.1, \mathrm{FDR}=0$ ) (Additional file 2; Figure S2a, S2b). MTOR Pathway $(\mathrm{NES}=1.79, \mathrm{FDR}=$ $0.0007)$ and $\mathrm{CD} 40$ Pathway (NES $=1.76, \mathrm{FDR}=0.0012)$ were also significantly enriched, which indicated a good agreement with our assumption.

\section{Immune infiltration analysis suggested TANs were influenced by PIK3CA expression}

UCEC immune infiltration status was calculated using TIMER, A Web Server for Comprehensive Analysis of Tumor-Infiltrating Immune Cells. As shown in Fig. 4, there was a significant increase in TANs in PIK3CA high group compare with PIK3CA low group $(P<0.0001)$, which was in good consistent with the KEGG enrichment analysis and GSEA. On the contrary, relative fraction of B cells, dendritic cells, CD4 T cells, CD8 T cells and macrophages showed no difference between two groups $(P>0.05)$. Our findings suggest that PIK3CA may play a key role in alteration of TANs in UCEC. And high TANs predicted poor prognosis in UCEC according to our analysis using TCGA UCEC clinical follow-up information $(p=0.09)$ (Additional file 3: Figure S3).
TANs related gene expression confirmed TANs modification by the influence of PIK3CA expression Finally, we verified the increase of TANs in PIK3CA high group by assessing TANs related gene expression. A cluster of TANs related genes expressed higher in PIK3CA high group, while the other cluster expressed higher in PIK3CA low group (Fig. 5a). Multiple TANs related gene expression formed a unique pattern in two groups, together to alteration the amount of TANs. TANs express several molecular markers [9], most of which express significantly differently between PIK3CA low group and PIK3CA high group, including ELANE (FDR = 0.0002), IL1B $($ FDR $=0.0009)$, ICAM1 (FDR < 0.0001), IL8 (FDR = 0.041), IL6 (FDR $=0.0027)$, CXCL2 $(F D R=0.0061)$, IRS1 $(\mathrm{FDR}<0.0001)$, CCL17 (FDR $=0.0019)$, PDGFRA (FDR $=$ 0.034), PDGFRL (FDR $<0.0001)$, OSMR (FDR $<0.0001)$, OSM (FDR $=0.011)($ Fig. 5b).

\section{Discussion}

UCEC is one of the most common cancer in female worldwide. Determining what factors cause UCEC progressing predominantly has been a subject of intense interest. Meanwhile, PIK3CA was proven to be a significant molecule during the progression of UCEC. Our result showed PIK3CA expressed differently, ranging from medium to high, in UCEC patients, which suggested tumor progression could be largely influenced PIK3CA expression quantity. PIK3CA, 


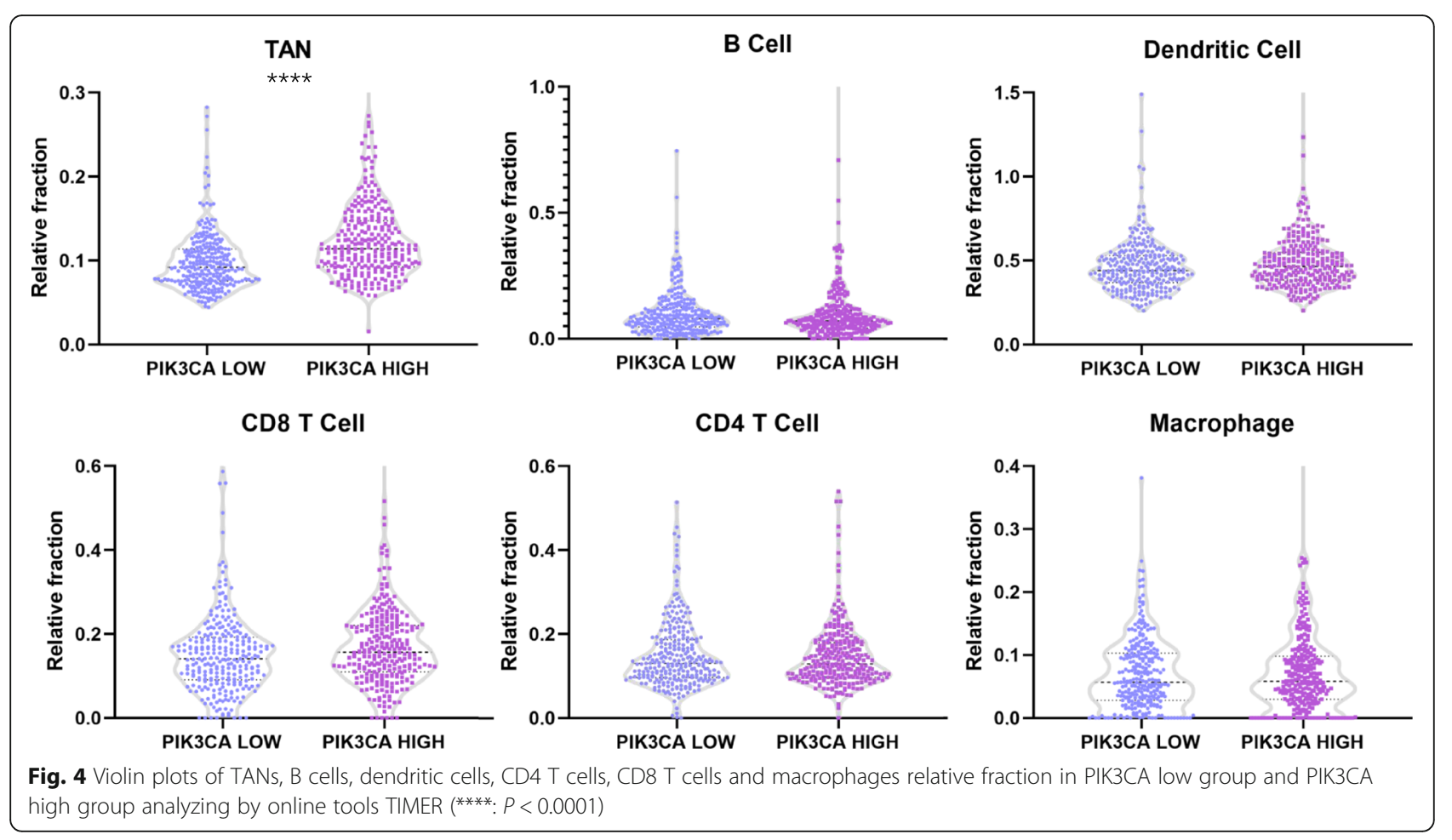

as expected, could change the outcome of UCEC patients, leading to shorter survival time in high PIK3CA group.

A lot of genes related to PIK3CA found in our research has been reported to correlate with other cancer types, which proved the importance of PIK3CA. PIK3R4 mutation was discovered in metastatic melanoma [22], suggested an important function in melanoma. Meanwhile, copy number gains of PIK3CA and PIK3R4 were associated with decreased survival in ovarian cancer [23]. PIK3R4 might alter PIK3CA expression in ovarian cancer.

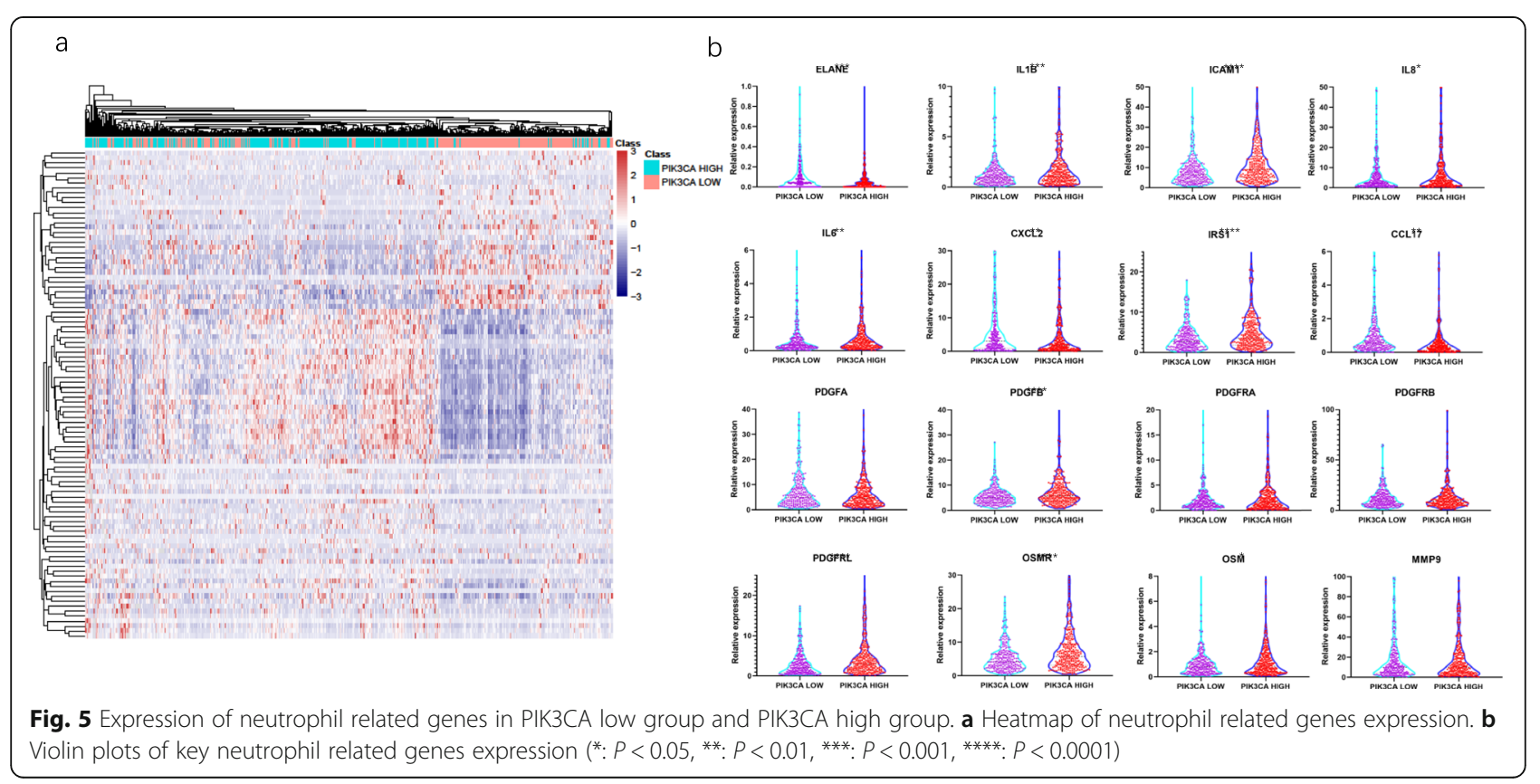


SENP2 have not been reported in UCEC related research. However, it may play a crucial role in hypoxia-induced lung cancer progression [24] and diagnosis or therapeutic targets for breast cancer treatment [25]. TBCCD1, a key regulator of centrosome positioning and consequently of internal cell organization, is a new centrosomal protein [26], and it has not been mentioned in any cancer.

Multiple kinds of cancer including thyroid cancer, prostate cancer, non-small cell lung cancer, glioma, colorectal cancer, chronic and acute myeloid leukemia, endometrial cancer and renal cell carcinoma seemed to affect by PIK3CA expression, some of which was demonstrated in a number of studies. PIK3CA may play a significant role in tumor cell proliferation, invasion, metastasis, apoptosis or cell cycle among different cancer types. It is worthwhile mentioning that PIK3CA probably regulates some common molecules or pathways in different cancer. Targeting PIK3CA to investigate and develop drugs may be a new way for better treatment of cancer, including UCEC.

Immunotherapy of cancer has become the center of attention these years. Most researches were related to $T$ cell or B cell, whereas few reports were related to TANs. However, TANs played an important part in immune microenvironment of many cancer types. It should be pointed out that no one has investigated TANs in UCEC. Our findings suggest that fraction of TANs are significantly altered by PIK3CA expression in UCEC, which is crucial for further studies of immune microenvironment and immunotherapy in UCEC.

The novelty of the study is that it was the first time and research to find out intrinsic mechanism in which PIK3CA influence TANs in UCEC. Our result implied the potential role of PIK3CA or other components of PI3K pathway in tumor immune microenvironment and may open a new approach for clinical anti-tumor research and anti-tumor drug development.

The shortcoming of our research was that we have not tested results in vivo owing to limitation of experimental conditions. However, we proposed a new aspect in UCEC immune infiltration status, which was influenced by PIK3CA expression.

Further effort is required to confirm the specific mechanism PIK3CA engaged to alter the fraction of TANs in UCEC. There is thereby an urgent need but it is still a significant challenge to uncover the role in immune system in UCEC.

\section{Method}

Acquisition and processing of TCGA UCEC patients' mRNA-seq data.

mRNA-seq FPKM data of TCGA UCEC patients was downloaded from The Cancer Genome Atlas. All UCEC patients were divided into two subgroups according to the expression of PIK3CA. PIK3CA low group contained 255 patients, whereas PIK3CA high group contained 256 patients. DEGs were calculated by setting $\log _{2}$ (Fold change) as \pm 1 and FDR as 0.01 . Utilizing ImageGP (http://www.ehbio.com/ImageGP/) drew Volcano plot. Heatmap was generated by $R$ (3.3.2) pheatmap package.

GO and KEGG enrichment analysis and GSEA.

Top 500 DEGs selected to process GO and KEGG enrichment analysis. GO and KEGG enrichment analysis were performed and visualized on website (http://enrich. shbio.com/index/ga.asp). Gene Set Enrichment Analysis (GSEA) is a computational method that determines whether an a priori defined set of genes shows statistically significant, concordant differences between two biological states. UCEC mRNA-seq data were divided into PIK3CA low group and PIK3CA high group As mentioned and performed GSEA. Several pathways were selected to analyze including Wnt Pathway, JNK MAPK Pathway, MTOR Pathway, CD40 Pathway using GSEA. NES and FDR were selected to value the pathway enrichment in each group. GSEA was performed using the GSEA 3.0 software.

\section{Immune infiltration analysis}

Immune infiltration status was analyzed by TIMER (https://cistrome.shinyapps.io/timer/), visualized by GraphPad Prism 8. We download TCGA tumor immune estimation data from website and filtered the UCEC PIK3CA low group and PIK3CA high group data for immune infiltration analysis. Percents of B cells, CD4 T cells, CD8 T cells, TANs, macrophages and dendritic cells in PIK3CA low group and PIK3CA high group were compared using $t$ test.

\section{Survival analysis}

Survival analysis of 542 TCGA UCEC patients was performed on Kaplan-Meier Survival Analysis [15] (http:// kmplot.com/analysis/). 542 patients were divided into two subgroups, PIK3CA low group $(n=213)$ and PIK3CA high group $(n=329)$. Survival of UCEC patients with high TANs and low TANs was illustrated with the help of TCGA UCEC clinical follow-up information.

\section{Immunohistochemical images acquisition}

Images of PIK3CA antibody stained UCEC tissue were obtained on Human Protein Atlas (https://www.proteinatlas. org/). The total number of UCEC patients screened for the analysis was 11 . The histological subtypes of analyzed pathological section were endometrium adenocarcinoma.

\section{cBioPortal}

One hundred seventy mutation sites in TCGA UCEC patients PIK3CA gene were analyzed by cBioPortal (http://www.cbioportal.org/), so as to survival status. 


\section{Additional files}

Additional file 1: Figure S1. GO and KEGG enrichment analysis of top 800 DEGs. (PDF 86 kb)

Additional file 2: Figure S2. GSEA analyzing PIK3CA low group and PIK3CA high group expression data in four pathways. (PDF 68 kb)

Additional file 3: Figure S3. Survival of UCEC patients with high TANs and low TANs. (PDF $10 \mathrm{~kb}$ )

\section{Abbreviations}

DEGs: Different Expressing Genes; GSEA: Gene Set Enrichment Analysis; TANs : Tumor Associated Neutrophils; TCGA: The Cancer Genome Atlas;

UCEC: Uterine Corpus Endometrial Carcinoma

\section{Acknowledgements}

We would like to thank the Department of Gynecology, The Second Affiliated Hospital of Hainan Medical University, Haikou, Hainan, China.

\section{Authors' contributions}

YP provided contribution to experimental design, data analysis, manuscript drafting and critical revising. $L J, Y L, Y H, Q D$ provided contribution to data acquisition, data processing, image painting, data analysis and interpretation. All authors have approved the final version and submission of this manuscript.

\section{Funding}

This study was supported by the Talent Scientic Research Foundation of Hainan Medical University.

\section{Availability of data and materials}

All mRNA-seq FPKM data is available in The Cancer Genome Atlas.

\section{Ethics approval and consent to participate}

Not applicable.

\section{Consent for publication}

Not applicable.

\section{Competing interests}

The authors declare that they have no competing interests.

\section{Author details}

'Department of Medical Oncology, Affiliated Hospital of Hainan Medica University, Haikou, Hainan 570102, People's Republic of China. ${ }^{2}$ Department of Gynecology, The Second Affiliated Hospital of Hainan Medical University, Haikou, Hainan 570102, People's Republic of China.

Received: 23 April 2019 Accepted: 20 August 2019

Published online: 31 August 2019

\section{References}

1. Matteson KA, Robison K, Jacoby VL. Opportunities for early detection of endometrial Cancer in women with postmenopausal bleeding. JAMA Intern Med. 2018;178(9):1222-3

2. Jemal A et al. Global cancer statistics. CA Cancer J Clin. 2011:61(2):69-90.

3. Dedes $\mathrm{K}$, et al. Emerging therapeutic targets in endometrial cancer. Nat Rev Clin Oncol. 2011;8(5):261-71.

4. Malentacchi F, et al. Pilot investigation of the mutation profile of PIK3CA/ PTEN genes (PI3K pathway) in grade 3 endometrial cancer. Oncol Rep. 2019; 41(3):1560-74.

5. Holst F, et al. PIK3CA amplification associates with aggressive phenotype but not markers of AKT-MTOR signaling in endometrial carcinoma. Clin Cancer Res. 2019;25(1):334-45.

6. Mclntyre JB, et al. PIK3CA missense mutation is associated with unfavorable outcome in grade 3 endometrioid carcinoma but not in serous endometrial carcinoma. Gynecol Oncol. 2014;132(1):188-93.

7. Konopka B, et al. PIK3CA mutations and amplification in endometrioid endometrial carcinomas: relation to other genetic defects and clinicopathologic status of the tumors. Hum Pathol. 2011;42(11):1710-9.
8. Kandoth $\mathrm{C}$, et al. Integrated genomic characterization of endometrial carcinoma. Nature. 2013:497(7447):67-73.

9. Hurt B, et al. Cancer-promoting mechanisms of tumor-associated neutrophils. Am J Surg. 2017;214(5):938-44.

10. Kim J, Bae JS. Tumor-associated macrophages and neutrophils in tumor microenvironment. Mediat Inflamm. 2016;2016:6058147.

11. Zhou SL, et al. Tumor-associated neutrophils recruit macrophages and Tregulatory cells to promote progression of hepatocellular carcinoma and resistance to Sorafenib. Gastroenterology. 2016;150(7):1646-1658.e17.

12. Gonzalez H, Hagerling C, Werb Z. Roles of the immune system in cancer: from tumor initiation to metastatic progression. Genes Dev. 2018;32(19-20): 1267-84.

13. Galdiero MR, et al. Occurrence and significance of tumor-associated neutrophils in patients with colorectal cancer. Int J Cancer. 2016;139(2):446-56.

14. Giese MA, Hind LE, Huttenlocher A. Neutrophil plasticity in the tumor microenvironment. Blood. 2019;133(20):2159-67.

15. Menyhart O, Nagy A, Gyorffy B. Determining consistent prognostic biomarkers of overall survival and vascular invasion in hepatocellular carcinoma. R Soc Open Sci. 2018;5(12):181006.

16. Gao J, et al. Integrative analysis of complex cancer genomics and clinical profiles using the cBioPortal. Sci Signal. 2013;6(269):pl1.

17. Cerami $E_{\text {, et al. The }}$ Bio cancer genomics portal: an open platform for exploring multidimensional cancer genomics data. Cancer Discov. 2012;2(5): 401-4.

18. Zhao J, et al. Prognostic value of KRAS/TP53/PIK3CA in non-small cell lung cancer. Oncol Lett. 2019:17(3):3233-40.

19. Zhou C, et al. A systems biology-based approach to uncovering molecular mechanisms underlying effects of traditional Chinese medicine Qingdai in chronic myelogenous leukemia, involving integration of network pharmacology and molecular docking technology. Med Sci Monit. 2018;24: 4305-16.

20. Christensen E, et al. Liquid biopsy analysis of FGFR3 and PIK3CA hotspot mutations for disease surveillance in bladder Cancer. Eur Urol. 2017:71(6): 961-9.

21. Yang L, Pang Y, Moses HL. TGF-beta and immune cells: an important regulatory axis in the tumor microenvironment and progression. Trends Immunol. 2010:31(6):220-7.

22. Shull AY, et al. Novel somatic mutations to PI3K pathway genes in metastatic melanoma. PLoS One. 2012;7(8):e43369.

23. Huang J, et al. Frequent genetic abnormalities of the PI3K AKT pathway in primary ovarian cancer predict patient outcome. Genes Chromosomes Cancer. 2011;50(8):606-18.

24. Hung PF, et al. Hypoxia-induced slug SUMOylation enhances lung cancer metastasis. J Exp Clin Cancer Res. 2019:38:5.

25. Heo KS. Regulation of post-translational modification in breast cancer treatment. BMB Rep. 2019:52(2):113-8.

26. Goncalves J, et al. TBCCD1, a new centrosomal protein, is required for centrosome and Golgi apparatus positioning. EMBO Rep. 2010;11(3):194-200.

\section{Publisher's Note}

Springer Nature remains neutral with regard to jurisdictional claims in published maps and institutional affiliations.

Ready to submit your research? Choose BMC and benefit from:

- fast, convenient online submission

- thorough peer review by experienced researchers in your field

- rapid publication on acceptance

- support for research data, including large and complex data types

- gold Open Access which fosters wider collaboration and increased citations

- maximum visibility for your research: over $100 \mathrm{M}$ website views per year

At BMC, research is always in progress.

Learn more biomedcentral.com/submissions 